\title{
Haikus on Materials Research
}

\author{
silicon carbide \\ with aluminum alloy- \\ stiff like winter's wind
}

David Weiss

Water droplets, cold

Crystals, dendrites, latent heat Snowflakes drift to earth

Julie Nucci

protein molecule self-assembled miracle functions in the folds

\section{E.N. Kaufmann}

Electrons and holes

Dancing in silicon chip

Like drops of spring rain

Robert W. Cahn

Midnight at the lab

My result is elusive

Engineers neglect

Drat! There goes my grant

Material properties

At their own peril

Julia Weertman and Paul Drzaic

Matt Ervin 